\title{
Environmental Policy for Non-Point Source Pollutions in a Bertrand Duopoly
}

\author{
Akio Matsumoto', Keiko Nakayama², Ferenc Szidarovszky ${ }^{3}$ \\ ${ }^{1}$ Department of Economics, Senior Researcher, International Center for further Development of Dynamic Economic Research, \\ Chuo University, Tokyo, Japan \\ ${ }^{2}$ Department of Economics, Chukyo University, Nagoya, Japan \\ ${ }^{3}$ Department of Applied Mathematics, University of Pécs, Pécs, Hungary \\ Email: akiom@tamacc.chuo-u.ac.jp, nakayama@mecl.chukyo-u.ac.jp, szidarka@gmail.com
}

How to cite this paper: Matsumoto, A., Nakayama, K. and Szidarovszky, F. (2018) Environmental Policy for Non-Point Source Pollutions in a Bertrand Duopoly. Theoretical Economics Letters, 8, 1058-1069. https://doi.org/10.4236/tel.2018.85073

Received: February 15, 2018

Accepted: April 14, 2018

Published: April 17, 2018

Copyright $\odot 2018$ by authors and Scientific Research Publishing Inc. This work is licensed under the Creative Commons Attribution International License (CC BY 4.0).

http://creativecommons.org/licenses/by/4.0/

\begin{abstract}
This study investigates the effectiveness of ambient charges under non-point source (NPS) pollutions in an imperfect competition framework. To this end, following Ganguli and Raju [1], it constructs a one-stage game and a two-stage game in which Bertrand duopolistic firms choose their best prices and abatement technology, respectively. It is demonstrated in both games that an increase in the ambient charge can lead to a decrease in pollution. This finding indicates that the ambient charge can efficiently control pollution in a Bertrand duopoly.
\end{abstract}

\section{Keywords}

Non-Point Source Pollution, Abatement Technology, Ambient Charge, Bertrand Competition, Two-Stage Game

\section{Introduction}

It is now well-known that most of today's pollutions such as air pollution, river pollution and lake pollution have multiple sources although any pollution originates from a single point source. It is, however, not known how to control these NPS (non-point source) pollutions. Segarson [2] suggests an ambient charge control: the government adopts an environmental policy to establish a cut-off level of the whole pollution and make the following rule, regardless of each firm's specific emission level, if the actual level of the total level exceeds the cut-off level, then all firms levy the same penalty while if the actual level falls short of the cut-off level, the all firms are awarded the same subsidy. The main purpose of this study is to demonstrate that the ambient charges can control the 
total amount of NPS pollutions under ála Bertrand imperfect competition. Based on the analysis of Raju and Ganguli [3] in a Cournot duopoly setting, Matsumoto et al. [4] show a "good-natured" effect of ambient charges in an $\mathrm{N}$-firm Cournot framework that an increase in the ambient charge leads to decreases of the total level of industry pollutions. On the other hand, Ganguli and Raju [1] consider the same subject in a Bertrand duopolistic market and numerically exhibit a "perverse" effect that an increase of ambient charge may lead to an increase in pollution in two distinct settings, one in the one-stage game and the other in the two-stage game. Ishikawa et al. [5] consider an ambient charge effect in an $\mathrm{N}$-firm Bertrand framework. This study shows the good-natured effect of ambient charges in Bertrand duopoly, analytically in one-stage game and numerically in two-stage game.

The rest of the paper is organized as follows. In section 2, the optimal price strategies of Bertrand duopolistic firms are derived in one-stage game in which all actions take place simultaneously. In Section 3, the optimal choices of abatement technology at the first stage and prices at the second stage are considered in two-stage game in which the actions take place in sequence. Concluding remarks and further extension of this study are given in Section 4.

\section{One-Stage Game}

In this section we consider the effect of the ambient charge in one stage game in which the regulator has announced the ambient charge and a cut-off ambient standard while two firms have fixed their pollution abatement technologies. Under this circumstance the firms choose their optimal prices to maximize their profits. Each firm produces a differentiated product. Market demand function for firm $i$ for $i, j=1,2$ and $i \neq j$ is

$$
q_{i}=a-p_{i}+\gamma p_{j}
$$

where $q_{i}$ denotes good $i$ produced by firm $i, p_{i}$ is the price of $q_{i}, p_{j}$ is the price for the good $j$ and $\gamma$ is a parameter with $0<\gamma<1$ measuring the substitutability between two goods. ${ }^{1}$ We exclude two extreme cases, one with $\gamma=1$ where the two goods are homogenous and the other with $\gamma=0$ where they are independent. The total amount of pollution $E$ generated by the two firms is given by

$$
E=\phi_{i} q_{i}+\phi_{j} q_{j}
$$

where $\phi_{i}$ and $\phi_{j}$ represent pollution abatement technologies of firms $i$ and j. $\phi_{i}=1$ means the worst technology with $100 \%$ pollution while $\phi_{i}=0$ means the best technology with no (0\%) pollution. Accordingly, it is assumed that $0 \leq \phi_{i} \leq 1$.

The profit function of firm $i$ is

${ }^{1}$ Intuitively, $x_{i}$ and $x_{j}$ are substitutes in the following sence that $\Delta p_{j}>0$ implies $\Delta x_{j}<0$ and $\Delta p_{j}>0$ with $\gamma>0$ implies $\Delta x_{i}>0$. Hence to a change in price $j$, the quantity response of good $i$ runs in an opposite direction of the response of good $j$. 


$$
\pi_{i}=p_{i} q_{i}-c q_{i}-t\left(\phi_{i} q_{i}+\phi_{j} q_{j}-\bar{E}\right)
$$

where $\bar{E}>0$ denotes ambient standard specified by the regulator, $c q_{i}$ is the production cost where $c>0$ is the common marginal cost of production and $t$ is an ambient charge or tax with $0 \leq t \leq 1$. According to the spirit of the ambient charge, although the two firms' contributions to pollutions might be different, each firm will pay the identical fine $t(E-\bar{E})$ if $E-\bar{E}>0$ and receive the identical subsidy $t(E-\bar{E})$ if $E-\bar{E}<0$. Substituting (1) into (3) and differentiating the resultant profit function with respect to $p_{i}$ give the first order condition for an interior solution maximizing profit of firm $i$,

$$
\frac{d \pi_{i}}{d p_{i}}=a-p_{i}+\gamma p_{j}+\left(p_{i}-c\right)(-1)-t\left[\phi_{i}(-1)+\phi_{j} \gamma\right]=0
$$

or

$$
2 p_{i}-\gamma p_{j}=a+c+t\left(\phi_{i}-\gamma \phi_{j}\right) .
$$

Maximizing $\pi_{j}$ with respect to $p_{j}$ presents a similar first-oder condition for firm $j$. Hence solving the following simultaneous system, which is obtained from first order conditions for firms $i$ and $j$,

$$
\left(\begin{array}{cc}
2 & -\gamma \\
-\gamma & 2
\end{array}\right)\left(\begin{array}{c}
p_{i} \\
p_{j}
\end{array}\right)=\left(\begin{array}{l}
a+c+t\left(\phi_{i}-\gamma \phi_{j}\right) \\
a+c+t\left(\phi_{j}-\gamma \phi_{i}\right)
\end{array}\right)
$$

yields the Bertrand equilibrium prices,

$$
\left(\begin{array}{c}
p_{i} \\
p_{j}
\end{array}\right)=\frac{1}{4-\gamma^{2}}\left(\begin{array}{ll}
2 & \gamma \\
\gamma & 2
\end{array}\right)\left(\begin{array}{l}
a+c+t\left(\phi_{i}-\gamma \phi_{j}\right) \\
a+c+t\left(\phi_{j}-\gamma \phi_{i}\right)
\end{array}\right)
$$

that are, after arranging the terms,

$$
\begin{aligned}
& p_{i}^{B}(\gamma, t)=\frac{1}{4-\gamma^{2}}\left\{(2+\gamma)(a+c)+t\left[\left(2-\gamma^{2}\right) \phi_{i}-\gamma \phi_{j}\right]\right\}, \\
& p_{j}^{B}(\gamma, t)=\frac{1}{4-\gamma^{2}}\left\{(2+\gamma)(a+c)+t\left[\left(2-\gamma^{2}\right) \phi_{j}-\gamma \phi_{i}\right]\right\} .
\end{aligned}
$$

Concerning the positivity of the Bertrand price, we have the following results.

Theorem 1 If $\phi_{i} \geq \phi_{j}$ or if $\phi_{i}<\phi_{j}$ and $a+c \geq 1 / 3$, then $p_{i}^{B}>0$.

Proof. If $\phi_{i} \geq \phi_{j}$ holds, then

$$
\begin{aligned}
& \left(2-\gamma^{2}\right) \phi_{i}-\gamma \phi_{j} \geq\left(2-\gamma^{2}-\gamma\right) \phi_{i} \\
& =(1-\gamma)(2+\gamma) \phi_{i}>0 .
\end{aligned}
$$

Then the first equation of (5) implies $p_{i}^{B}>0$. Now suppose that $\phi_{i}<\phi_{j}$. If the right hand side of the first equation of (5) is equal to zero, then solving it for $\phi_{j}$ gives the form of

$$
\phi_{j}=\frac{2-\gamma^{2}}{\gamma} \phi_{i}+\frac{(2+\gamma)(a+c)}{\gamma t} .
$$

Assumption $t \leq 1$ implies 


$$
\frac{(2+\gamma)(a+c)}{\gamma t} \geq \frac{(2+\gamma)(a+c)}{\gamma} .
$$

Since $\gamma /(2+\gamma)<1 / 3$ for $\gamma<1$, the term on the right hand side is greater than unity if $a+c \geq 1 / 3$ under which, for all $\phi_{j} \leq 1$,

$$
\phi_{j}<\frac{2-\gamma^{2}}{\gamma} \phi_{i}+\frac{(2+\gamma)(a+c)}{\gamma t} \text {. }
$$

The inequality implies that $p_{i}^{B}>0$.

Q.E.D.

Differentiating the Bertrand price of firm $i$ with respect to $t$ reveals that the sign of the derivative is the same as the sign of the terms in the square brackets in (5),

$$
\operatorname{sign}\left[\frac{d p_{i}^{B}}{d t}\right]=\operatorname{sign}\left[\left(2-\gamma^{2}\right) \phi_{i}-\gamma \phi_{j}\right]
$$

Hence the effect caused by a change in the ambient charge on the Bertrand prices can summarized as follows.

Theorem 2 A firm with a larger or equal abatement technology positively responds to a change in the ambient charge whereas the response of a firm with a smaller abatement technology is ambiguous,

$$
\begin{aligned}
& \text { If } \phi_{i}>\phi_{j} \text {, then } \frac{d p_{i}^{B}}{d t}>0 \text { and } \frac{d p_{j}^{B}}{d t} \lesseqgtr 0, \\
& \text { If } \phi_{i}=\phi_{j} \text {, then } \frac{d p_{i}^{B}}{d t}>0 \text { and } \frac{d p_{j}^{B}}{d t}>0, \\
& \text { If } \phi_{i}<\phi_{j} \text {, then } \frac{d p_{i}^{B}}{d t} \lesseqgtr 0 \text { and } \frac{d p_{j}^{B}}{d t}>0 .
\end{aligned}
$$

Proof. If $\phi_{i} \geq \phi_{j}$, then for firm $i$, the bracketed terms in (5) are

$$
\left(2-\gamma^{2}\right) \phi_{i}-\gamma \phi_{j}>(2+\gamma)(1-\gamma) \phi_{i}>0 \text { implying that } \frac{d p_{i}^{B}}{d t}>0
$$

and for firm $j$, from the bracketed terms in the second equation of (5) to be equal to zero, we can define the ratio of the abatement technologies

$$
\left(\frac{\phi_{j}}{\phi_{i}}\right)^{*}=\frac{\gamma}{2-\gamma^{2}} \text {. }
$$

This ratio is less than unity implying

$$
\frac{d p_{j}^{B}}{d t} \lesseqgtr 0 \text { according to } \frac{\phi_{j}}{\phi_{i}} \lesseqgtr\left(\frac{\phi_{j}}{\phi_{i}}\right)^{*} \text { when } \frac{\phi_{j}}{\phi_{i}} \leq 1 \text {. }
$$

The same procedure can be applied for the case of $\phi_{i}<\phi_{j}$.

Substituting the Bertrand prices into the demand functions in (1) presents the Bertrand outputs of firm $i$ and $j$,

$$
\begin{aligned}
& q_{i}^{B}=a-p_{i}^{B}+\gamma p_{j}^{B}, \\
& q_{j}^{B}=a-p_{j}^{B}+\gamma p_{i}^{B} .
\end{aligned}
$$


To check whether $q_{i}^{B}$ is positive, we subtract the second equation of (6) from the first equation to obtain

$$
q_{i}^{B}-q_{j}^{B}=(1+\gamma)\left(p_{j}^{B}-p_{i}^{B}\right)
$$

where, in the same way, from (5)

$$
p_{i}^{B}-p_{j}^{B}=\frac{1+\gamma}{2+\gamma} t\left(\phi_{i}-\phi_{j}\right) .
$$

Concerning the positivity of the Bertrand output, we have the following:

Theorem 3 If $a \geq c+1$, then $q_{i}^{B}>0$ and $q_{j}^{B}>0$ for $0 \leq \phi_{k} \leq 1$ for $k=1,2$.

Proof. If $\phi_{i} \geq \phi_{j}$ holds, then (8) leads to $p_{i}^{B} \geq p_{j}^{B}$ with which (7) implies $q_{i}^{B} \leq q_{j}^{B}$. On the other hand, the first equation of (6) with the forms of $p_{i}^{B}$ and $p_{j}^{B}$ given in (5) is reduced to

$$
q_{i}^{B}=\frac{a_{3} \gamma^{3}+a_{2} \gamma^{2}+a_{1} \gamma+a_{0}}{-\left(4-\gamma^{2}\right)}
$$

where

$$
\begin{gathered}
a_{3}=t \phi_{j}>0, \\
a_{2}=-c<0, \\
a_{1}=-\left(a+c+3 t \phi_{j}\right)<0, \\
a_{0}=-2\left(a-c-t \phi_{i}\right) \leq 0
\end{gathered}
$$

The direction of the last inequality is due to $a \geq c+1$ and $t \phi_{i} \leq 1$. Let the numerator of (9) be $f(\gamma)$. Then due to Descartes' rule of sign, $f(\gamma)=0$ has only one positive root, $\gamma_{0}>0$. Substituting $\gamma=1$ gives

$$
f(1)=a_{3}+a_{2}+a_{1}+a_{0}=-2\left(a+t \phi_{j}\right)-\left(a-t \phi_{i}\right)<0 .
$$

The last inequality means that $f(\gamma)<0=f\left(\gamma_{0}\right)$ for $\gamma<1$, with which then (9) leads to $q_{i}^{B}>0$, implying that $q_{j}^{B}>0$ as well. If $\phi_{j}>\phi_{i}$, then interchanging the two firms generates the same result.

Q.E.D.

Now consider the effect of a change in the ambient charge on each output level.

$$
\begin{aligned}
& \frac{d q_{i}^{B}}{d t}=-\frac{d p_{i}^{B}}{d t}+\gamma \frac{d p_{j}^{B}}{d t}=\frac{1}{4-\gamma^{2}}\left[-2 \phi_{i}+\gamma\left(3-\gamma^{2}\right) \phi_{j}\right], \\
& \frac{d q_{j}^{B}}{d t}=-\frac{d p_{j}^{B}}{d t}+\gamma \frac{d p_{i}^{B}}{d t}=\frac{1}{4-\gamma^{2}}\left[-2 \phi_{j}+\gamma\left(3-\gamma^{2}\right) \phi_{i}\right] .
\end{aligned}
$$

Concerning the ambient charge effect on output, we have the following results.

Theorem 4 A firm with a larger or equal abatement technology negatively responds to a change in the ambient charge whereas the response of a firm with a smaller abatement technology is ambiguous. 


$$
\begin{aligned}
& \text { If } \phi_{i}>\phi_{j} \text {, then } \frac{d q_{i}^{B}}{d t}<0 \text { and } \frac{d q_{j}^{B}}{d t} \lesseqgtr 0 \text {, } \\
& \text { If } \phi_{i}=\phi_{j} \text {, then } \frac{d q_{i}^{B}}{d t}<0 \text { and } \frac{d q_{j}^{B}}{d t}<0 \text {, } \\
& \text { If } \phi_{i}<\phi_{j} \text {, then } \frac{d q_{i}^{B}}{d t} \lesseqgtr 0 \text { and } \frac{d q_{j}^{B}}{d t}<0 \text {. }
\end{aligned}
$$

Proof. If $\phi_{i}=\phi_{j}$, then $\gamma\left(3-\gamma^{2}\right)<2$ for $\gamma<1$ immediately implies the results. If $\phi_{i}>\phi_{j}$, then $\gamma\left(3-\gamma^{2}\right)>0$ for $\gamma>0$ indicates that for firm $i$,

$$
-2 \phi_{i}+\gamma\left(3-\gamma^{2}\right) \phi_{j}<\left[-2+\gamma\left(3-\gamma^{2}\right)\right] \phi_{i}<0 \text { implying } \frac{d q_{i}^{B}}{d t}<0
$$

and for firm $j$, there is a threshold value of the parameter ratio such as

$$
\frac{\gamma\left(3-\gamma^{2}\right)}{2}=\left(\frac{\phi_{j}}{\phi_{i}}\right)^{*}<1 \text { leading to } \frac{d q_{j}^{B}}{d t} \gtreqless 0 \text { according to } \frac{\phi_{j}}{\phi_{i}} \lesseqgtr\left(\frac{\phi_{j}}{\phi_{i}}\right)^{*} \text { and } \frac{\phi_{j}}{\phi_{i}}<1 \text {. }
$$

The same procedure can be applied for the case of $\phi_{i}<\phi_{j}$.

Q.E.D.

It should be noticed first that firm $i$ with a larger $\phi_{i}$ has an inefficient technology because it generates larger emission. Theorems 2 and 4 imply that, the firm with inefficient technology exhibits natural response to the change in the ambient charge, that is, it increases price and decreases output. On the other hand the firm with efficient technology responds ambiguously. It should be noticed second that these firm-specific responses are non-observable for the regulator which can see only the total amount in the case of NPS pollution.

The total level of pollution at the Bertrand equilibrium is obtained by substituting $q_{i}^{B}$ and $q_{j}^{B}$ into (2)

$$
E^{B}=\phi_{i} q_{i}^{B}+\phi_{j} q_{j}^{B}
$$

Concerning the effect of a change in the ambient charge on the total pollution, we have the following result.

Theorem 5 An increase in the ambient charge decreases the total level of pollution,

$$
\frac{d E^{B}}{d t}<0
$$

Proof. Differentiating $E^{B}$ with respect $t$ gives

$$
\frac{d E^{B}}{d t}=\phi_{i} \frac{d q_{i}^{B}}{d t}+\phi_{j} \frac{d q_{j}^{B}}{d t}=\frac{-2}{4-\gamma^{2}}\left\{\phi_{i}^{2}+\phi_{j}^{2}-\gamma\left(3-\gamma^{2}\right) \phi_{i} \phi_{j}\right\}
$$

Notice that $\gamma\left(3-\gamma^{2}\right) \leq 2$ and the equality holds when $\gamma=1$. Hence

$$
\phi_{i}^{2}+\phi_{j}^{2}-\gamma\left(3-\gamma^{2}\right) \phi_{i} \phi_{j}>\phi_{i}^{2}+\phi_{j}^{2}-2 \phi_{i} \phi_{j}=\left(\phi_{i}-\phi_{j}\right)^{2} \geq 0 \text {. }
$$

Therefore we arrive at the result where the strict inequality is due to the assumption, $\gamma<1$.

Q.E.D.

Although Theorem 4 implies a possibility of the perverse effect on emission of the individual firm with the efficient abetment technology, Theorem 5 implies 
that the total effect is always negative, implying that the negative effect of the inefficient firm dominates the positive effect of the efficient firm.

\section{Two-Stage Game}

In this section the firms and the regulator take actions in two stages. At the first stage, each firm determines its optimal abatement technology whereas the regulator announces the ambient charge and the cut-off level of total pollution. Then at the second stage, the firms choose their prices to maximize their profits, given the ambient charge, the cut-of level and their abatement technologies.

The decision-making at the second stage have been already considered in the one-stage game. Given the Bertrand prices and outputs in (5) and (6), we consider the actions of choosing abatement technology at the first stage. The Bertrand profit function of firm $i$ under Bertrand prices and Bertrand output is defined as

$$
\pi_{i}^{B}=p_{i}^{B} q_{i}^{B}-\left[c q_{i}^{B}+\left(1-\phi_{i}\right)^{2}\right]-t\left(\phi_{i} q_{i}^{B}+\phi_{j} q_{j}^{B}-\bar{E}\right)
$$

for $i, j=1,2$ and $i \neq j$. Notice that there is a small difference between the definitions of (3) and (11). There is a term $\left(1-\phi_{i}\right)^{2}$ in (11) and no such a term in (3). This term reflects the cost associated with selecting the abatement technology. ${ }^{2}$ At the second stage, the abatement technology has been already selected somehow and thus it does not affect the determination of the optimal price whether this cost is included or not. However this cost function is effective for choosing technology at the first stage where the firm $i$ determines its abatement technology, $\phi_{i}$, so as to maximize its Bertrand profit. Differentiating (11) with respect to $\phi_{i}$ gives the first-order condition for the optimal level of $\phi_{i}$,

$$
\frac{\partial \pi_{i}^{B}}{\partial \phi_{i}}=\frac{\partial \pi_{i}^{B}}{\partial p_{i}} \frac{\partial p_{i}^{B}}{\partial \phi_{i}}+\frac{\partial \pi_{i}^{B}}{\partial p_{j}} \frac{\partial p_{j}^{B}}{\partial \phi_{i}}+\left.\frac{\partial \pi_{i}^{B}}{\partial \phi_{i}}\right|_{p_{i}^{B}, p_{j}^{B} ; \text { given }}=0
$$

where

$$
\begin{aligned}
& \frac{\partial \pi_{i}^{B}}{\partial p_{i}}=0 \text { by the first order condition at the second stage, } \\
& \frac{\partial \pi_{i}^{B}}{\partial p_{j}}=\gamma p_{i}^{B}-c \gamma-t\left(\gamma \phi_{i}-\phi_{j}\right) \\
& \left.\frac{\partial \pi_{i}^{B}}{\partial \phi_{i}}\right|_{p_{i}^{B}, p_{j}^{B} ; \text { given }}=2\left(1-\phi_{i}\right)-t\left(a-p_{i}^{B}+\gamma p_{j}^{B}\right) \\
& \frac{\partial p_{j}^{B}}{\partial \phi_{i}}=-\frac{\gamma t}{4-\gamma^{2}} .
\end{aligned}
$$

Arranging the terms in $\partial \pi_{i}^{B} / \partial \phi_{i}=0$ with taking account of the forms of the Bertrand prices in (5) yield the modified FOC at the first stage,

$$
2\left[(2 t)^{2}-\left(4-\gamma^{2}\right)^{2}\right] \phi_{i}-\left(\gamma^{4}-9 \gamma^{2}+16\right) \gamma t^{2} \phi_{j}=-2\left(4-\gamma^{2}\right)^{2}+t d
$$

${ }^{2}$ See Raju and Ganguli [3] for this cost. 
where

$$
d=4(2+\gamma)[a-(1-\gamma) c]
$$

In the same way, arranging the terms in $\partial \pi_{j}^{B} / \partial \phi_{j}=0$ yields the modified FOC for firm $j$

$$
-\left(\gamma^{4}-9 \gamma^{2}+16\right) \gamma t^{2} \phi_{i}+2\left[(2 t)^{2}-\left(4-\gamma^{2}\right)^{2}\right] \phi_{j}=-2\left(4-\gamma^{2}\right)^{2}+t d .
$$

Solving (12) and (13) simultaneously for $\phi_{i}$ and $\phi_{j}$ presents the optimal abatement technology for both firms,

$$
\phi_{i}^{*}=\phi_{j}^{*}=\phi^{*}(\gamma, t)=\frac{-2\left(4-\gamma^{2}\right)^{2}+t d}{2\left[(2 t)^{2}-\left(4-\gamma^{2}\right)^{2}\right]-\left(\gamma^{4}-9 \gamma^{2}+16\right) \gamma t^{2}} .
$$

Although the form of the solution seems to be highly complicated, the following result is obtained.

Proposition 1 If $2 / 3 \geq a>c$, then the optimal abatement technology $\phi^{*}(\gamma, t)$ is positive for $0<\gamma<1$ and $0 \leq t \leq 1$.

Proof. It is numerically confirmed that the denominator of (14) is negative for $0<\gamma<1$ and $0 \leq t \leq 1 .{ }^{3}$ The numerator is rewritten as

$$
2(2+\gamma)\{2[a-(1-\gamma) c] t-f(\gamma)\}
$$

where

$$
f(\gamma)=(2-\gamma)^{2}(2+\gamma) \text { and } 3<f(\gamma)<8 \text { for } 0<\gamma<1 .
$$

Assumption $a>c$ implies $a-(1-\gamma) c>0$. The sign of the terms in the braces of $(15)$ is negative for $t=0$ (i.e., $-f(\gamma)<0$ ) and is also negative for $t=1$ if $2 a<3$. As the terms satisfies the inequality

$$
2[a-(1-\gamma) c]-f(\gamma)<2 a-f(\gamma) .
$$

and the lower bound of $f(\gamma)$ is 3 , then the right hand side is negative for $0<\gamma<1$ if $2 a \leq 3$. Thus the sign of the left hand side is also negative. Hence $2[a-(1-\gamma) c] t-f(\gamma)<0$ for $0 \leq t \leq 1$. Therefore (14) with the negative denominator and the negative numerator implies $\phi^{*}(\gamma, t)>0$.

Q.E.D.

Notice that the following set is not empty,

$$
\{(a, c) \mid 0<a \leq 3 / 2 \text { and } 0<c \leq a-1\} \text {, }
$$

implying that the conditions imposed on the values of $a$ and $c$ given in Theorem 3 and Proposition 1 are compatible. Substituting $\phi^{*}$ into the Bertrand prices in (5) gives the optimal Bertrand price

$$
p_{i}^{*}=p_{j}^{*}=p^{*}(\gamma, t)=\frac{1}{2-\gamma}\left[(a+c)+(1-\gamma) t \phi^{*}(t)\right]
$$

that is clearly positive for $0<\gamma<1$ and $0 \leq t \leq 1$, which is summarized as follows.

${ }^{3}$ Numerical calculations of this and any others that follow are done with Mathematica, ver. 11. 
Proposition 2 If $3 / 2 \geq a>c$, then the optimal Bertrand price $p^{*}(\gamma, t)$ is positive for $0<\gamma<1$ and $0 \leq t \leq 1$.

The optimal Bertrand output is obtained by substituting $p^{*}(\gamma, t)$ into the demand function, (1),

$$
q^{*}(\gamma, t)=a-(1-\gamma) p^{*}(\gamma, t) .
$$

Concerning the optimal output, we can have the following,

Proposition $3 q^{*}(\gamma, t)<0$ for $\gamma=0$ and $t=1$.

Proof. Substituting $\gamma=0$ into (14) and (16) present

$$
p^{*}(0, t)=\frac{1}{2}\left[(a+c)+t \frac{t(a-c)-4}{2\left(t^{2}-1\right)}\right]
$$

that is, in turn, substituted into (17) to obtain

$$
q^{*}(0, t)=\frac{1}{4\left(t^{2}-1\right)}\left[3(a-c) t^{2}+4 t-2(a-c)\right]
$$

then

$$
\lim _{t \rightarrow 1, t<1} q^{*}(0, t)=\lim _{t \rightarrow 1, t<1} \frac{a-c+4}{4\left(t^{2}-1\right)}<0
$$

where the numerator is positive and the denominator is negative. This completes the proof.

Q.E.D.

Proposition 3 implies that $q^{*}(\gamma, t)$ is inevitably negative for a small neighborhood of point $(0,1)$. Numerical confirmation of Proposition 3 is given in Figure 1 in which the Bertrand output is negative in the shaded region and positive otherwise. ${ }^{4}$ The shaded region should be eliminated from further considerations.



Figure $1, \mathrm{q}^{*}>0$ in the white region and $\mathrm{q}^{*}<0$ in the shaded region.

${ }^{4} a=3 / 2$ and $c=1 / 2$ are taken for this and the following examples. The vertical and horisontal intersecpts of the black curve are

$$
\gamma_{0} \simeq 0.187 \text { and } t_{0} \simeq 0.563 \text {. }
$$


Finally, the optimal level of total pollution is

$$
E^{*}(\gamma, t)=2 \phi(\gamma, t) q^{*}(\gamma, t)
$$

that is apparently negative if $q^{*}(\gamma, t)<0$, that is, in the shaded region in Figure 1. Its derivative with respect to $t$ is

$$
\frac{d E^{*}}{d t}=2 \frac{q^{*} \phi^{*}}{t}\left[\frac{t}{\phi^{*}} \frac{\partial \phi^{*}}{\partial t}+\frac{t}{q^{*}} \frac{\partial q^{*}}{\partial t}\right]
$$

where $\partial \phi^{*} / \partial t$ and $\partial q^{*} / \partial t$ can be of either sign and thus the sign of the terms in the square bracket seems ambiguous. Numerically, as as shown in Figure 2(a) and Figure 2(b), each derivative is confirmed to be positive in the corresponding shaded region in which

$$
\hat{\gamma}_{0} \simeq 0.160 \text { and } \hat{t}_{0} \simeq 0.53 \text { in Figure 2(a) }
$$

and

$$
\bar{\gamma}_{0} \simeq 0.594 \text { and } \bar{t}_{0}=0.5 \text { in Figure 2(b). }
$$

The dotted upward-sloping curve in Figure 2(a) is described by the $q^{*}=0$ curve. The shaded region is included in the region with $q^{*}<0$ so that the derivative of the optimal technology is negative in the feasible region with $q^{*}>0$. Hence the sign of the tax-derivative of the total emission level is definitely negative in the white region in Figure 2(b) as both derivatives are negative. On the other hand, it is sensitive to the relative magnitude between the elasticities of the optimal ambient technology and the optimal output with respect to $t$ in the shaded region in Figure 2(b).

Given the parameter values, Figure 3 illustrates the values of $d E^{*} / d t$ against a pair of $(\gamma, t)$ with $0<\gamma<1$ and $0 \leq t \leq 1$. Since it is seen that any point on the $3 \mathrm{D}$ surface in Figure 3 is negative, it is numerically shown that $d E^{*} / d t<0$ for $0<\gamma<1$ and $0 \leq t \leq 1$. According to equation (19), the sign of $d E^{*} / d t$ is

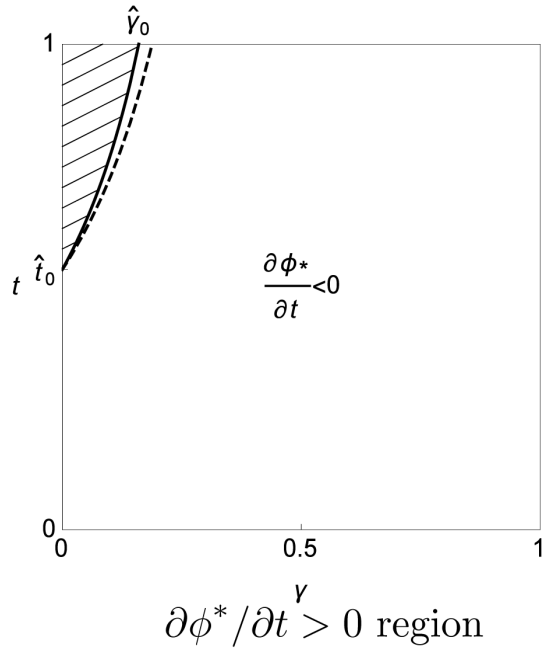

(a)

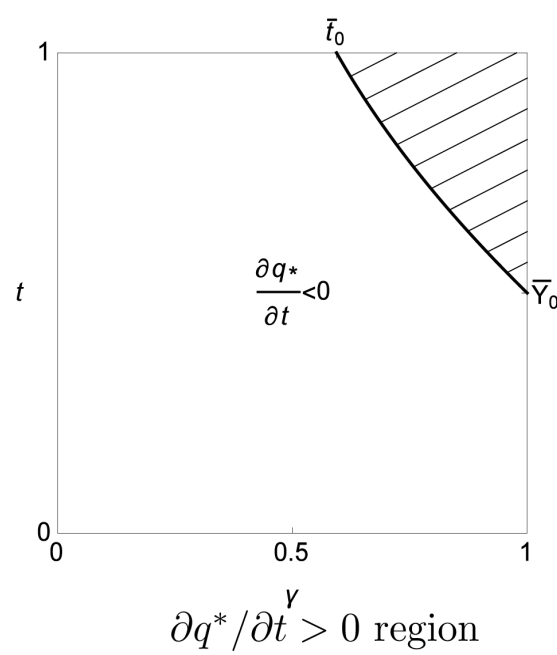

(b)

Figure 2. Divisions of the nonnegative $(\gamma, t)$ region. 


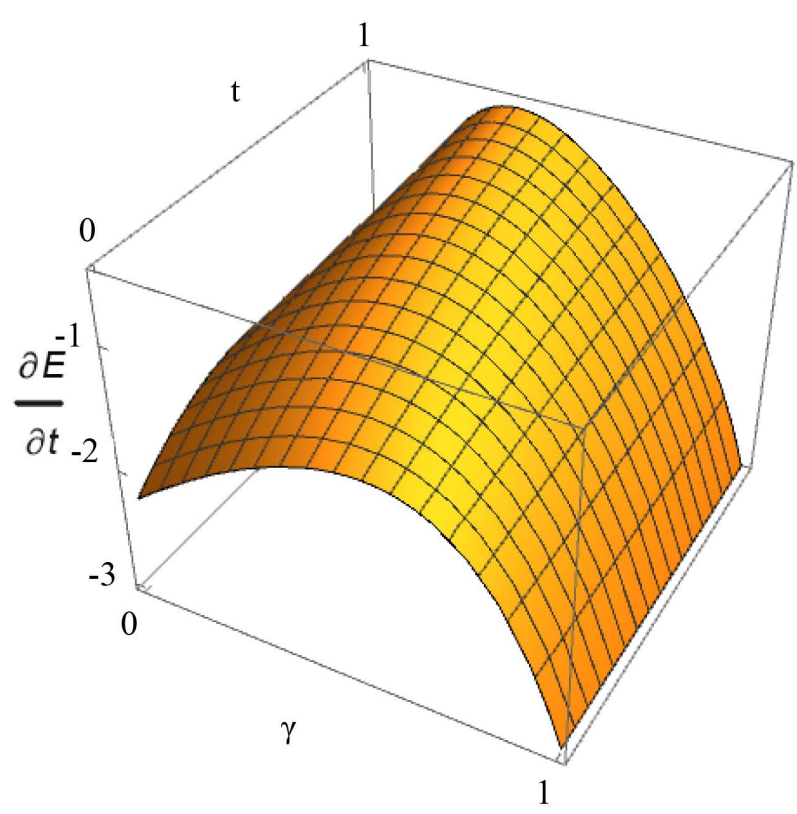

Figure 3. Effective ambient charge under $a=3 / 2$ and $c=1 / 2$.

ambiguous in the shaded region of Figure 2(b). However, Figure 3 implies that the elasticity of $\phi^{*}$ in absolute value is larger than that of $q^{*}$, leading to the negative sign of $d E^{*} / d t$. Therefore we have our main result that an increase in ambient charge always decreases the total level of optimal pollution. ${ }^{5} \mathrm{We}$ summarize this result.

Proposition 4 It is numerically confirmed that a change in the ambient charge has the good-natured effect,

$$
\frac{d E^{*}}{d t}<0 \text { for } 0<\gamma<1 \text { and } 0 \leq t \leq 1 .
$$

\section{Concluding Remark}

In this paper, we reconsider the "perverse" effect caused by a change in ambient charges shown by Ganguli and Raju [1]. To this end, following their basic framework, we first re-examine the effect in one-stage game in which the Bertrand firms determine their prices so as to maximize their profits, given the abatement technology. Our first result analytically demonstrates that an increase of ambient charges decreases the total level of NPS pollutions. We then turn attention to the effect in two-stage game in which the optimal abatement technology is selected at the first stage and the optimal prices are determined at the second stage. Our second result numerically shows the good-natured effect on the total level of pollution. With these results, we conclude that the ambient charge might be an efficient method to control NPS pollutions even in a duopoly Bertrand market.

${ }^{5}$ We obtain this results with various values of $a$ and $c$. However, we are unable to prove it analytically so this is an numerically-shown result. 


\section{Funding}

The authors highly acknowledge the financial supports from the MEXT-Supported Program for the Strategic Research Foundation at Private Universities 2013-2017, the Japan Society for the Promotion of Science (Grant-in-Aid for Scientific Research (C), 16K03556, 18K01631 and 17H01931) and Chuo University (Joint Research Grant). The usual disclaimer applies.

\section{References}

[1] Ganguli, S. and Raju, S. (2012) Perverse Environmental Effects of Ambient Charges in a Bertrand Duopoly. Journal of Environmental Economics and Policy, 1, 289-296. https://doi.org/10.1080/21606544.2012.714972

[2] Segerson, K. (1988) Uncertainty and Incentives for Non-Point Pollution Control. Journal of Environmental Economics and Management, 15, 87-98. https://doi.org/10.1016/0095-0696(88)90030-7

[3] Raju, S. and Ganguli, S. (2013) Strategic Firm Interaction, Return to Scale, Environmental Regulation and Ambient Charges in a Cournot Duopoly. Technology and Investment, 4, 113-122. https://doi.org/10.4236/ti.2013.42014

[4] Matsumoto, A., Szidarovszky, F. and Yabuta, M. (2018) Environmental Effects of Ambient Charge in Cournot Oligopoly. Journal of Environmental Economic Policy, 7, 41-56. https://doi.org/10.1080/21606544.2017.1347527

[5] Ishikawa, T., Matsumoto, A. and Szidarovszky, F. (2017) Regulation of Non-Point Source Pollution under n-Firm Bertrand Competition. IERCU DP \#284, Institute of Economic Research, Chuo University. 